\title{
Efecto del entrenamiento en pretemporada en las variables antropométricas y derivadas en jugadores de baloncesto de élite \\ Effect of preseason training on anthropometric and derived variables in professional basketball players \\ Mario Albaladejo, Raquel Vaquero-Cristóbal, Francisco Esparza-Ros \\ Universidad Católica de Murcia (España)
}

\begin{abstract}
Resumen. El objetivo de este estudio fue analizar los efectos provocados por un periodo de entrenamiento en la pretemporada sobre las variables antropométricas y derivadas en jugadores de baloncesto de élite. En la presente investigación participaron ocho jugadores profesionales de la liga ACB, con una edad de 26,8 $\pm 5,4$ años. Se realizaron dos valoraciones cineantropométricas aplicando los criterios establecidos por la International Society for the Advancement of Kynanthropometry. La primera medición (pre-test) se realizó justo antes de comenzar la pretemporada, mientras que la segunda medición (post-test) fue realizada 14 días después. Se encontró una disminución significativa del peso, el pliegue de la cresta ilíaca, abdominal y muslo, el perímetro de la cintura, el sumatorio de seis y ocho pliegues, la ratio cintura/cadera y en el porcentaje y peso graso. También se halló un aumento significativo del porcentaje de masa muscular. En conclusión, se ha hallado que catorce días de pretemporada son suficientes para provocar cambios en algunos parámetros antropométricos, sobre todo los relacionados con la adiposidad, así como en la composición corporal, más concretamente sobre la masa grasa y muscular. Palabras clave: Cineantropometría, composición corporal, deporte, proporcionalidad, somatotipo.
\end{abstract}

Abstract. The aim of this study was to analyze the effects of a preseason training focused on anthropometric and derived variables in elite basketball players. Eight professional players from ACB, with a mean age of 26.8 \pm 5.4 years old, participated in this study. Anthropometric evaluations were taken according with the criteria of the International Society for the Advancement of Kynanthropometry. The first test was done before the preseason began (pre-test), meanwhile the second one was taken 14 days after (post-test). Significant decreases were found in weight, iliac crest, abdominal and thigh skinfolds, waist girth, six and eight skinfold thickness sums, waist/hip ratio, and fat percentage and mass. Also, muscle mass significantly increased. In conclusion, fourteen preseason training days are sufficient to produce changes in some anthropometric parameters, especially those related to adiposity, as well as in body composition, specifically in fat and muscle mass.

Key words: Body composition, kynanthropometry, proportionality, somatotype, sport.

\section{Introducción}

Existen diferentes factores que pueden influir sobre el rendimiento deportivo (Hoare, 2000). Uno de los aspectos con mayor relevancia son las características morfológicas del deportista, encontrándose que los atletas de élite de cada modalidad deportiva presentan una composición corporal y unos aspectos morfológicos similares entre ellos y diferentes a los que caracterizan a los atletas de otras modalidades (Guedes, 2012). Por lo tanto, la optimización de las variables antropométricas y derivadas resulta clave para poder mejorar el rendimiento deportivo (Esparza-Ros, 1993).

El baloncesto es un deporte de contacto físico donde predominan las acciones rápidas y explosivas, combinando sprints con saltos y cambios de dirección (Ramos, Rubio, Martínez, Esteban \& Jiménez, 2010). Fruto de estas características particulares, es necesario tener una serie de capacidades tanto físicas como psicológicas para obtener un rendimiento óptimo en este deporte (Torres-Unda, Zarrazquin, Gil, Ruiz, Irazusta, Kortajarena, Seco \& Irazusta, 2013). Estudios previos han relacionado el rendimiento en baloncesto con determinadas variables antropométricas tales como el peso, la talla, el porcentaje de grasa y de músculo o el índice de masa corporal (IMC) (Abella del Campo, Escortell, Sospedra, Norte-Navarro, Martínez-Rodríguez \& MartinezSanz, 2015; Del Pozo, Olcina, Brazo, Robles \& Muñoz, 2009; Godoy-Cumillaf, Cárcamo-Araneda, Hermosilla-Rodríguez y

Fecha recepción: 07-11-18. Fecha de aceptación: 26-05-19 Raquel Vaquero-Cristóbal

rvaquero@ucam.edu
Viveros-Herrera 2015; Jelicic, Sekulic \& Marinovic, 2002; Tárrega \& Canda, 2010). En esta línea, en estudios realizados con jugadores de baloncesto de categoría cadete y junior se ha encontrado que el somatotipo varía ligeramente entre los diferentes jugadores, si bien los componentes predominantes suelen ser la ectomorfia y la mesomorfia (Abella del Campo et al., 2015; Jelicic et al., 2002).

Varios autores han analizado las diferencias entre jugadores de baloncesto y jugadores de otros deportes de equipo en cuanto a altura, peso, IMC y porcentaje de grasa, aspectos relevantes en el rendimiento atendiendo a las características propias del baloncesto (Gaurav, Singh \& Singh, 2010; Popovic, Akpinar, Jaksic, Matic \& Bjelica., 2013; Ziv \& Lidor, 2009). En estos estudios se observó que los jugadores de baloncesto presentaban un mayor peso y una mayor altura respecto a jugadores de voleibol y de fútbol, pero sin encontrar diferencias significativas en el IMC. Los jugadores de baloncesto obtuvieron también mayores valores en el porcentaje graso que los jugadores de los deportes anteriormente mencionados (Gaurav et al., 2010; Popovic et al., 2013). Al ser comparados con el modelo phantom, jugadores de élite mexicanos mostraron valores $\mathrm{z}$ negativos en peso y pliegues cutáneos, así como valores positivos en diámetros óseos y los perímetros del brazo relajado y contraído, indicando estos resultados que los jugadores de baloncesto poseen una adiposidad menor y un desarrollo óseo y muscular superior a la media establecida por este modelo (Rivera-Sosa, Martínez \& Ortiz-Gómez, 2017).

Los parámetros antropométricos pueden variar con el crecimiento o el estilo de vida, de tal manera que una práctica 
deportiva sistematizada y una especialización deportiva podría inducir cambios en las variables antropométricas (Rivera-Sosa, 2016). Al respecto, algunas variables antropométricas y derivadas de los jugadores cambia durante el transcurso de la temporada debido a factores como la alimentación o la carga que suponen los entrenamientos y la competición. Diversos autores defienden que en otros deportes de equipo, como el futbol, el periodo de tiempo donde mayores cambios se pueden observar es en la pretemporada y los primeros compases de la temporada competitiva, debido a la mayor intensidad y volumen de los entrenamientos (Carling \& Orhant, 2010; Ostojic, 2003). Resultados similares hallaron Fuke, Dal Pupo y Corrêa (2009), encontrando que tras una pretemporada de cuatro semanas se produjeron cambios significativos en el porcentaje graso y en la masa corporal en futbolistas profesionales.

No obstante, no se han encontrado estudios que hayan analizado los cambios que se producen en pretemporada en las variables antropométricas de los jugadores de baloncesto, menos aún en jugadores profesionales. Por lo tanto, el objetivo de este estudio fue analizar los efectos provocados por un periodo de entrenamiento en la pretemporada sobre las variables antropométricas y derivadas en jugadores de baloncesto de élite.

\section{Método}

\section{Participantes}

Ocho jugadores de baloncesto de élite participaron voluntariamente en este estudio (media de edad 26,8+5,4 años). Todos ellos pertenecientes a un equipo masculino de la liga ACB que además jugaba competiciones europeas.

\section{Procedimiento}

Previo a la realización del proyecto, se obtuvo la aprobación del comité de ética institucional y los participantes firmaron un consentimiento informado.

Se realizaron dos valoraciones cineantropométricas aplicando los criterios establecidos por la International Society for the Advancement of Kynanthropometry (ISAK) (Stewart, Marfell-Jones, Olds, \& de Ridder, 2011). Un antropometrista criterio, nivel 4 acreditado por la ISAK, realizó la valoración antropométrica a todos los participantes.

La primera medición (pre-test) se realizó justo antes de comenzar la pretemporada, mientras que la segunda medición (post-test) fue realizada 14 días después. Entre las dos mediciones los deportistas que formaban la muestra realizaron dos entrenamientos diarios, uno en sesión de mañana y otro en sesión de tarde, seis días a la semana. Las cargas en estos entrenamientos fueron individualizadas por el equipo técnico de acuerdo a las características individuales de cada jugador.

Se valoraron las medidas básicas de peso, talla y envergadura; los pliegues del tríceps, subescapular, bíceps, supraespinal, abdominal, muslo y pierna; los perímetros del brazo relajado, brazo contraído y flexionado, cintura, cadera y pierna; y los diámetros óseos del húmero, biestiloideo y del fémur. Cada medida se tomó dos o tres veces dependiendo de si la diferencia entre las dos primeras medidas era superior al $5 \%$ en los pliegues cutáneos y al 1\% en el resto de las medidas. Una vez realizadas las medidas correspondientes se realizó la media en caso de haber necesitado dos mediciones o la mediana en caso de que se hubieran realizado tres, como valor definitivo para su análisis posterior.

Las mediciones tanto el pre-test como el post-test se realizaron en las mismas condiciones de temperatura $\left(25^{\circ} \mathrm{C}\right)$ y sin haber realizado los participantes ejercicio físico ni comidas pesadas las 24 horas antes de la toma de datos.

Posteriormente se calculó el IMC, dividiendo el peso en kilogramos entre la altura en metros al cuadrado; los sumatorios de seis y ocho pliegues, estando en el primero recogidos todos los pliegues menos el del bíceps y la cresta iliaca; los perímetros del brazo y de la pierna corregidos (perímetro corregido = perímetro $-\Pi x$ pliegue); el área muscular transversal de la pierna y el brazo (área muscular transversal $\left.=[\text { perímetro }- \text { pliegue } \mathrm{x} \Pi]^{\wedge} 2 / 4 \Pi\right)$; $\mathrm{y}$ la ratio cintura-cadera (perímetro cintura / perímetro cadera). Siguiendo el modelo de cuatro componentes de Rose \& Guimaraes (1980), se valoró el porcentaje de masa grasa (Yuhasz, 1974), peso muscular (Matiegka, 1921), porcentaje de masa ósea (Rocha, 1975) y peso residual (Würch 1974). Para hallar el somatotipo se siguió la estrategia de Carter y Heath (1990), obteniéndose el valor de endomorfia, mesomorfia y ectomorfia. Por último, para obtener información acerca de las variables de proporcionalidad de los jugadores se utilizó la estrategia del modelo Phantom (Ross y Wilson, 1974).

\section{Instrumentación}

El material utilizado para medir la masa corporal fue una báscula SECA 862 (SECA, Alemania) de 100 g de precisión. Para la talla se empleó un antropómetro GPM (GPM, Suiza) con una precisión de 0,1cm. Los pliegues cutáneos se midieron con un plicómetro Holtain (Holtain Ltd., Reino Unido) de 0,2 mm de precisión. Para los perímetros y la envergadura se empleó una cinta métrica milimetrada inextensible Lufkin W606PM (Lufkin, E.E.U.U.). Los díámetros fueron valorados con un paquímetro Holtain (Holtain Ltd., Reino Unido) de 0,1 mm de precisión. Con el objetivo de eliminar posibles errores en la toma de datos, el material fue calibrado antes de las mediciones realizadas.

\section{Análisis estadístico}

Para la realización del análisis estadístico se valoró la distribución de la muestra mediante el test de normalidad de Shapiro-Wilk. Para los datos que siguieron una distribución normal se realizó un análisis basado en pruebas paramétricas. En el caso de las variables que no siguieron una distribución normal, pliegue del muslo, porcentaje de masa grasa y valor $\mathrm{Z}$ del pliegue del muslo en el pre-test; y pliegue del tríceps, bíceps, supraespinal, muslo y pierna , sumatorio de seis y ocho pliegues, los valores Z de los pliegues del tríceps, bíceps, supraespinal y muslo y la masa grasa en el post-test, se utilizaron pruebas no paramétricas. Mediante la realización de estadística descriptiva se obtuvieron los valores medios y la desviación típica de las diferentes variables. Para determinar las diferencias entre el pre- y post-test se utilizó una prueba $t$-student para variables relacionadas con las variables paramétricas y la prueba de Wilcoxon para las variables no paramétricas, considerando las diferencias como estadísticamente significativas cuando $p<0,05$. Todo el aná- 
lisis se realizó con el paquete estadístico SPSS (versión 21.0). También se calculó el tamaño del efecto mediante la prueba $d$ de Cohen con el programa Microsoft $\subset$ Excel XP (Microsoft Corporation, EE.UU.). Un valor menor de 0,2 se consideró como tamaño de efecto bajo; un valor entre 0,2 y 0,4 un tamaño del efecto bajo-moderado; un valor entre 0,4 y 0,6 un tamaño del efecto moderado; un valor entre 0,6 y 0,8 un tamaño del efecto moderado-alto; y un valor superior a 0,8 un tamaño del efecto alto (28).

\section{Resultados}

En la Tabla 1 se puede observar la media y la desviación típica obtenida para cada una de las variables antropométricas en el pre-test y el post-test. También se puede encontrar la significación estadística de la diferencia entre ambas mediciones.

Se encontró una disminución significativa del peso; pliegue de la cresta ilíaca, abdominal y muslo; perímetro de la cintura; sumatorio de seis y ocho pliegues; ratio cintura/ cadera; y porcentaje y peso de masa grasa. También se halló un aumento significativo del porcentaje de masa muscular. Todas estas variables mostraron un tamaño del efecto entre bajo y moderado-alto, obteniendo el estadístico $d$ de Cohen valores entre 0,07 y 0,67 (Tabla 1). No se encontraron diferencias significativas en el resto de medidas incluidas en la valoración. No obstante, como se puede observar en el perfil de pliegues (Figura I), se produjo una tendencia a la disminución de los mismos tras el periodo de entrenamiento.

Respecto al somatotipo, los jugadores mostraron un somatotipo mesomorfo balanceado en ambas mediciones. En la primera medida se encontró una marcada tendencia hacia la mesomorfia y valores similares de endomorfia y

\begin{tabular}{|c|c|c|c|c|}
\hline Variable & Pre-test & Post-test & Valor de $t$ y $p$ & d de Cohon \\
\hline Peso & $98,59 \pm 15,36$ & $97,57 \pm 14,61$ & $t=2,43 ; p=0,045$ & $\mathbf{0 , 0 7}$ \\
\hline Talla & $198,41 \pm 12,02$ & $198,41 \pm 11,94$ & $t=-1,00 ; p=0,951$ & 0,00 \\
\hline Envergadura & $204,92 \pm 12,64$ & $204,96 \pm 12,84$ & $t=1,14 ; p=0,865$ & 0,00 \\
\hline PL tríceps* & $11,91 \pm 4,25$ & $10,84 \pm 3,51$ & $t=-1,12 ; p=0,262$ & 0,28 \\
\hline PL subescapular & $10,55 \pm 2,10$ & $10,12 \pm 1,78$ & $t=1,14 ; p=0,292$ & 0,22 \\
\hline PL bíceps* & $4,69 \pm 0,81$ & $4,57 \pm 0,93$ & $t=-0,84 ; p=0,399$ & 0,13 \\
\hline PL cresta ilíaca & $17,29 \pm 4,36$ & $14,57 \pm 3,76$ & $t=4,87 ; p=0,002$ & 0,67 \\
\hline PL supraespinal* & $11,27 \pm 4,16$ & $10,21 \pm 3,19$ & $t=-1,68 ; p=0,093$ & 0,29 \\
\hline PL abdominal & $22,15 \pm 6,89$ & $20,17 \pm 6,19$ & $t=3,24 ; p=0,014$ & 0,30 \\
\hline PL muslo* & $13,34 \pm 5,84$ & $11,92 \pm 5,50$ & $t=-2,53 ; p=0,012$ & 0,25 \\
\hline PL pierna medial* & $8,35 \pm 3,08$ & $8,00 \pm 2,53$ & $t=1,04 ; p=0,334$ & 0,12 \\
\hline PR brazo relajado & $34,08 \pm 2,18$ & $33,89 \pm 2,09$ & $t=2,76 ; p=0,445$ & 0,09 \\
\hline PR brazo contraído & $36,61 \pm 2,43$ & $36,68 \pm 2,31$ & $t=-0,47 ; p=0,654$ & 0,03 \\
\hline PR cintura & $87,93 \pm 4,80$ & $85,95 \pm 4,29$ & $t=6,46 ; p>0,001$ & 0,44 \\
\hline PR cadera & $105,51 \pm 6,84$ & $104,68 \pm 5,83$ & $t=1,29 ; p=0,238$ & 0,13 \\
\hline PR pierna & $40,67 \pm 3,17$ & $40,59 \pm 3,02$ & $t=0,98 ; p=0,360$ & 0,03 \\
\hline D húmero & $7,84 \pm 0,37$ & $7,84 \pm 0,38$ & $t=0,80 ; \mathrm{p}=0,985$ & 0,00 \\
\hline D biestiloideo & $6,05 \pm 0,23$ & $6,05 \pm 0,21$ & $t=0,95 ; p=0,875$ & 0,00 \\
\hline $\mathrm{D}$ fémur & $10,58 \pm 0,43$ & $10,58 \pm 0,49$ & $t=1,07 ; \mathrm{p}=0,901$ & 0,00 \\
\hline PR brazo corregido & $30,34 \pm 2,06$ & $30,49 \pm 1,89$ & $t=-0,55 ; p=0,600$ & 0,08 \\
\hline PR pierna corregido & $38,04 \pm 3,23$ & $38,07 \pm 3,03$ & $t=-0,24 ; p=0,817$ & 0,01 \\
\hline AMT brazo & $73,54 \pm 9,98$ & $74,22 \pm 9,10$ & $t=-0,51 ; p=0,626$ & 0,07 \\
\hline AMT pierna & $115,91 \pm 19,58$ & $116,00 \pm 18,43$ & $t=-0,12 ; p=0,904$ & 0,00 \\
\hline IMC & $24,93 \pm 2,13$ & $24,69 \pm 2,04$ & $t=2,10 ; p=0,074$ & 0,11 \\
\hline Sumatorio 6 PL* & $77,57 \pm 22,00$ & $71,27 \pm 20,14$ & $t=-2,52 ; p=0,012$ & 0,30 \\
\hline Sumatorio 8 PL* & $99,55 \pm 26,49$ & $90,42 \pm 24,55$ & $t=-2,52 ; p=0,012$ & 0,36 \\
\hline Ratio cintura/cadera & $0,83 \pm 0,03$ & $0,82 \pm 0,26$ & $t=2,71 ; p=0,03$ & 0,44 \\
\hline Índice ponderal & $43,06 \pm 1,35$ & $43,19 \pm 0,74$ & $t=-1,95 ; p=0,091$ & 0,00 \\
\hline Envergadura relativa & $1,03 \pm 2,88$ & $1,03 \pm 2,76$ & $t=0,12 ; p=0,897$ & 0,00 \\
\hline \% grasa* & $11,16 \pm 2,13$ & $10,55 \pm 1,95$ & $t=-2,52 ; p=0,012$ & 0,30 \\
\hline Peso graso* & $11,15 \pm 3,38$ & $10,42 \pm 3,10$ & $t=-2,38 ; p=0,017$ & 0,22 \\
\hline \% Masa ósea & $16,03 \pm 1,13$ & $16,18 \pm 1,05$ & $t=-1,81 ; p=0,113$ & 0,13 \\
\hline Peso óseo & $15,70 \pm 1,90$ & $15,70 \pm 1,87$ & $t=-1,00 ; p=0,851$ & 0,00 \\
\hline$\%$ masa residual & $24,10 \pm 0,00$ & $24,10 \pm 0,00$ & $t=0,00 ; p=1,000$ & 0,82 \\
\hline Peso residual & $23,76 \pm 3,70$ & $23,52 \pm 3,52$ & $t=2,43 ; p=0,654$ & 0,07 \\
\hline$\%$ masa muscular & $48,70 \pm 1,58$ & $49,17 \pm 1,52$ & $t=-4,53 ; p=0,003$ & 0,30 \\
\hline Peso muscular & $47,98 \pm 7,49$ & $47,94 \pm 7,13$ & $t=0,22 ; p=0,835$ & 0,01 \\
\hline Endomorfia & $2,91 \pm 0,74$ & $2,68 \pm 0,61$ & $t=2,04 ; p=0,081$ & 0,34 \\
\hline Mesomorfia & $4,67 \pm 1,05$ & $4,69 \pm 1,07$ & $t=-0,67 ; p=0,523$ & 0,02 \\
\hline Ectomorfia & $2,94 \pm 0,98$ & $3,04 \pm 1,02$ & $t=-1,88 ; p=0,101$ & 0,10 \\
\hline
\end{tabular}

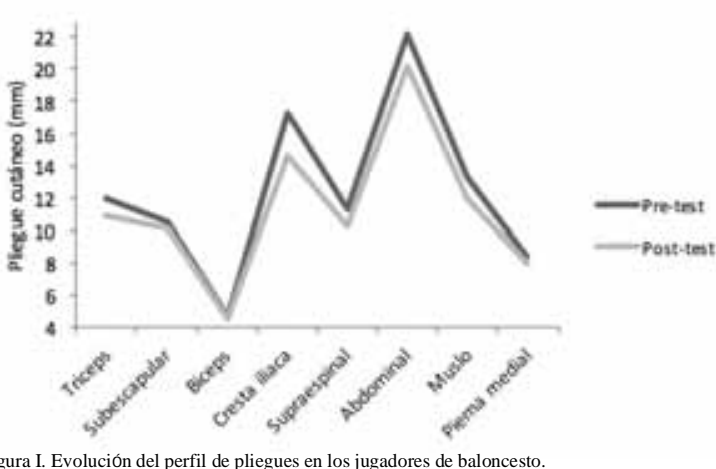

Figura I. Evolución del perfil de pliegues en los jugadores de baloncesto.

ectomorfia, mientras que en la segunda medición se mantuvo el valor de la mesomorfia, disminuyendo el valor de la endomorfia y aumentando el de la ectomorfia (Tabla 1). Como consecuencia de esto se produjo un pequeño cambio del somatopunto (Figura II).

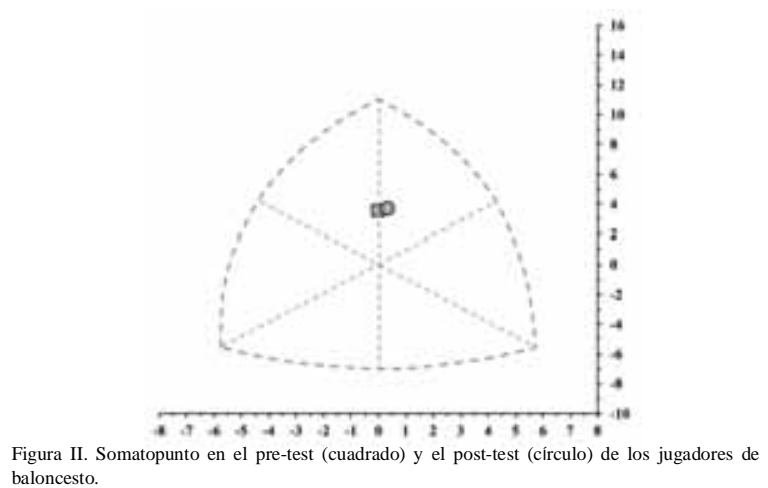

En la Tabla 2 se puede encontrar la media y desviación típica y la comparación de los valores $Z$ correspondientes a las dos mediciones. Los jugadores mostraron un valor $Z$ positivo para la envergadura y los perímetros corregidos. En cambio, el valor fue negativo para el peso, los pliegues cutáneos y el porcentaje graso. Se encontraron diferencias significativas en el valor $Z$ del pliegue de la cresta ilíaca, del abdominal y del muslo, el perímetro del brazo relajado y porcentaje de grasa, no siendo significativas las diferencias entre el resto de las medidas analizadas. Todas estas variables mostraron un tamaño del efecto entre bajo y moderado-alto, obteniendo el estadístico $d$ de Cohen valores entre 0,11 y 0,76 (Tabla 2).

Tabla 2.

\begin{tabular}{|c|c|c|c|c|}
\hline & & & & \\
\hline Variable & Pre-test & Post-test & Valor de $t$ y $p$ & $d$ de Cohen \\
\hline $\mathrm{Z}$ peso & $-0,29 \pm 0,71$ & $-0,36 \pm 0,72$ & $t=1,93 ; p=0,095$ & 0,09 \\
\hline $\mathrm{Z}$ envergadura & $0,47 \pm 0,66$ & $0,47 \pm 0,66$ & $t=-0,53 ; p=0,891$ & 0,00 \\
\hline Z PL tríceps* & $-1,18 \pm 0,72$ & $-1,38 \pm 0,57$ & $t=-1,12 ; p=0,263$ & 0,31 \\
\hline Z PL subescapular & $-1,61 \pm 0,33$ & $-1,68 \pm 0,31$ & $t=1,05 ; p=0,330$ & 0,21 \\
\hline Z PL bíceps* & $-1,99 \pm 0,29$ & $-2,04 \pm 0,33$ & $t=-0,94 ; p=0,345$ & 0,16 \\
\hline Z PL cresta ilíaca & $-1,12 \pm 0,49$ & $-1,46 \pm 0,40$ & $t=4,71 ; p=0,002$ & 0,76 \\
\hline Z PL supraespinal* & $-1,29 \pm 0,73$ & $-1,49 \pm 0,54$ & $t=-1,68 ; p=0,093$ & 0,31 \\
\hline Z PL abdominal & $-0,82 \pm 0,77$ & $-1,05 \pm 0,66$ & $t=3,11 ; p=0,017$ & 0,31 \\
\hline Z PL muslo* & $-1,87 \pm 0,55$ & $-2,02 \pm 0,51$ & $t=-2,52 ; \mathrm{p}=0,012$ & 0,28 \\
\hline Z PL pierna medial & $-1,90 \pm 0,50$ & $-1,96 \pm 0,41$ & $t=0,99 ; p=0,356$ & 0,13 \\
\hline Z PR brazo relajado & $1,01 \pm 0,56$ & $0,95 \pm 0,58$ & $t=2,79 ; p=0,027$ & 0,11 \\
\hline Z PR brazo contraído & $0,84 \pm 0,56$ & $0,87 \pm 0,58$ & $t=-0,50 ; p=0,634$ & 0,05 \\
\hline Z PR cintura & $0,82 \pm 0,92$ & $0,44 \pm 0,92$ & $t=6,89 ; p<0,001$ & 0,41 \\
\hline Z PR cadera & $-0,74 \pm 0,92$ & $-0,86 \pm 0,60$ & $t=1,30 ; p=0,233$ & 0,19 \\
\hline Z PR pierna & $-0,15 \pm 0,91$ & $-0,18 \pm 0,92$ & $t=0,88 ; p=0,410$ & 0,03 \\
\hline Z D húmero & $0,73 \pm 0,77$ & $0,73 \pm 0,77$ & $t=0,23 ; p=0,875$ & 0,00 \\
\hline Z D biestiloideo & $-0,03 \pm 0,97$ & $-0,03 \pm 0,97$ & $-t=0,27 ; p=0,881$ & 0,00 \\
\hline Z D fémur & $-0,89 \pm 0,97$ & $-0,89 \pm 0,97$ & $t=0,36 ; p=0,785$ & 0,00 \\
\hline $\mathrm{Z}$ brazo corregido & $3,24 \pm 0,75$ & $3,20 \pm 0,75$ & $t=0,67 ; p=0,522$ & 0,05 \\
\hline Z pierna corregido & $2,01 \pm 1,12$ & $2,00 \pm 1,11$ & $t=0,48 ; p=0,645$ & 0,02 \\
\hline $\mathrm{Z} \%$ grasa & $-1,44 \pm 0,74$ & $-1,59 \pm 0,44$ & $t=4,25 ; p=0,004$ & 0,34 \\
\hline
\end{tabular}




\section{Discusión}

El objetivo de este estudio fue determinar si tras un corto periodo de entrenamiento en la pretemporada se producen cambios significativos en las variables antropométricas y derivadas en jugadores de baloncesto de élite. El principal hallazgo de esta investigación fue que se produjo una disminución de aquellas variables relacionadas con la adiposidad tales como el pliegue de la cresta ilíaca, el pliegue abdominal y muslo; los sumatorios de seis y ocho pliegues; y la masa y porcentaje de grasa. También se encontró una disminución de la masa corporal y un aumento del porcentaje de masa muscular de los jugadores tras el periodo de observación. La modificación en la composición corporal mostrada por los jugadores de baloncesto del presente estudio coincide con los datos de estudios anteriores realizados en futbolistas, en los que se encontró que la composición corporal se modifica rápidamente en el periodo de la pretemporada, produciéndose la mayoría de los cambios en la masa grasa (Carling, \& Orhant, 2010; Fuke et al., 2009; Ostojic, 2003).

La reducción de la masa grasa es un factor de gran importancia en el entrenamiento debido a su relación con el rendimiento deportivo. Concretamente en baloncesto, se ha encontrado una relación inversa entre la potencia del salto y la masa grasa (Puentes \& Díaz, 2016). Los cambios en las variables relacionadas con la adiposidad podrían estar causados por la carga de entrenamiento a la que fueron sometidos los jugadores que participaron en la presente investigación, pues realizaron durante la pretemporada dos sesiones diarias durante seis días a la semana. Este entrenamiento pudo haber provocado un balance energético negativo, que es un factor determinante en la pérdida de grasa corporal y en consecuencia, potenciar la disminución de las variables relacionadas con la adiposidad (Vargas, Lancheros \& Barrera, 2011). Estudios previos ya han encontrado que los jugadores de baloncesto pueden ver reducido su porcentaje de grasa y aumentado su porcentaje muscular con la práctica sistemática de este deporte (Siders, Bolonchuk \& Lukaski, 1991) como consecuencia de las demandas específicas del deporte en cuanto al entrenamiento y a las capacidades físicas. Al ser analizado el sumatorio de seis pliegues cutáneos en cinco categorías de jugadores profesionales se observó que no había diferencias entre ellas, y que los valores se situaban entre 60 y $80 \mathrm{~mm}$ (Vaquera et al., 2015). Al inicio del estudio, los participantes obtuvieron un valor de 77,57 mm, situándose próximos al límite superior descrito en la literatura, lo que podría estar influido por que venían de un periodo de desentrenamiento; mientras que en la medición correspondiente al post-test el valor obtenido fue de 71,27 mm, reduciéndose significativamente y aproximándose al valor central respecto a la literatura. El porcentaje de grasa ya se encontraba por debajo de los valores descritos para jugadores de la liga ACB en el pre-test, mostrando un $11,16 \%$, frente al $13 \%$ obtenido en otros estudios (Vaquera et al., 2015). A pesar de esto, el porcentaje de grasa se redujo de forma significativa, hecho de relevancia debido a su estrecha relación con el rendimiento en baloncesto (Puentes \& Díaz, 2016).

La masa muscular es de vital importancia en el rendimiento deportivo y en baloncesto cobra gran relevancia tanto en las acciones ofensivas como defensivas, cuando los juga- dores deben mantener su posición frente a los rivales (Vaquera et al., 2015). Sobre el aumento significativo del porcentaje muscular tras el periodo de observación, la evolución de esta variable es consecuencia de que se produjo un descenso significativo de la masa corporal total del individuo, sin que hubiera cambios en la masa muscular. Estudios previos han apuntado a que un gran balance energético negativo podría implicar una disminución incluso de la masa muscular (Garthe, Raastad, Refsnes, Koivisto \& Sundgot-Borgen, 2011). No obstante, un entrenamiento correctamente planificado, junto con una dieta equilibrada y suficiente, podría provocar que incluso en situaciones de déficit calórico se produzca un mantenimiento de la masa muscular (Helms, Aragon, Fitschen, 2014). Resultados similares se han encontrado tras un periodo de pretemporada en jugadores de fútbol (Carling \& Orhant, 2010; Fuke et al., 2009; Ostojic, 2003). No se vieron modificados ni los perímetros corregidos ni las áreas musculares transversales, variables también relacionadas con el desarrollo muscular del deportista, lo que podría ser consecuencia del corto periodo transcurrido entre ambas mediciones. Sería conveniente en futuros estudios analizar la evolución de estas variables en periodos algo superiores de tiempo.

La masa corporal es uno de los parámetros que influyen en el rendimiento deportivo mostrando una relación inversa, ya que una menor masa corporal se asocia a un mejor desempeño en el deporte, según lo descrito en la literatura (Hoffman, Lebus, Ganong, Casazza, \& Loan, 2010; Tárrega \& Canda, 2010). Debido al mantenimiento de masa muscular y la pérdida de masa grasa, la masa corporal sufrió una disminución significativa tras la pretemporada. Estos cambios pueden estar basados en el tipo de entrenamiento realizado durante la pretemporada, habiéndose observado que el ejercicio orientado a la mejora de la potencia y la capacidad aeróbica son los que más influencia tienen en la pérdida de masa corporal (Campos, González-Villora, González-Gómez \& Martins, 2018), siendo estas capacidades fundamentales en la práctica del baloncesto (Ramos et al., 2010). Al ser comparados con jugadores del mismo nivel competitivo se puede observar que los participantes de este estudio se encontraban por encima en peso en la medición del pre-test (Vaquera et al., 2015). Tras el periodo de entrenamiento transcurrido, los valores en esta variable se aproximaron a los descritos en la literatura previa.

La disminución de la ratio cintura/cadera tiene una más que contrastada importancia para la salud, siendo un indicador del descenso de riesgo de sufrir enfermedades cardiovasculares (Latorre, Salas \& Soto-Hermoso, 2012; Terry, Wood, Haskell, Stefanick \& Krauss, 1989). Por otro lado, la disminución de porcentaje y masa grasa del tronco se ha relacionado con un mejor rendimiento deportivo (Hoffman et al., 2010).En este estudio se observó que el perímetro de la cintura disminuyó de forma significativa, disminuyendo de esta manera también la ratio cintura/cadera al no producirse modificaciones en el perímetro de la cadera. Estos cambios podrían estar relacionados con la disminución del porcentaje y la masa grasa en el tronco, tal y como se demuestra al analizar los pliegues cutáneos.

El somatotipo no se modificó significativamente, si bien sí que se encontró una predominancia de la mesomorfia y una tendencia a la disminución de la endomorfia y al aumen- 
to de la ectomorfia tras el entrenamiento. Esto podría acercar el somatotipo de los jugadores al encontrado en estudios previos realizados en jugadores de baloncesto, en los que se encuentra una predominancia del componente mesomórfico sobre la ectomorfia y la endomorfia, siendo este último el componente con valores menores (Abella del Campo et al., 2015; Jelicic, Sekulic \& Marinovic, 2002). En otros deportes de equipo, como en el fútbol, también se ha observado que cuanto mayor es el nivel competitivo, mas tendente a la mesomorfia es el somatotipo (Zuñiga-Galaviz, OsorioGutiérrez, Toledo-Domínguez \& Herrera-Perea, 2017).

Respecto a los valores de proporcionalidad, los jugadores mostraron una mayor envergadura y perímetros corregidos, indicadores estos últimos del desarrollo músculo-esquelético, de lo establecido por el modelo Phantom. Por el contrario, en los pliegues cutáneos y el porcentaje graso mostraron valores inferiores a los encontrados en el modelo, a pesar de encontrarse en pretemporada. El modelo Phantom ha sido empleado con anterioridad en jugadores de baloncesto, demostrando su utilidad como herramienta para comparar las características antropométricas en deportistas (Rivera-Sosa et al., 2017). Los resultados encontrados en este estudio se asemejan a los encontrados por Rivera-Sosa et al. (2017) en jugadores de baloncesto mexicanos de diferentes niveles competitivos.

La ausencia de cambios en la talla, envergadura, envergadura relativa, los diámetros y el porcentaje óseo y residual podría deberse a que los sujetos eran adultos, siendo necesario en esta etapa grandes periodos de tiempo para que estas variables se modifiquen significativamente (Cabañas et al., 2008). Al no producirse cambios en la talla y tener los cambios en el peso corporal un tamaño del efecto leve, el IMC y el índice ponderal tampoco se vieron alterados (Esparza-Ros, 1993). La altura de la muestra fue superior a la de los jugadores descritos por Vaquera et al. (2015), lo que podría deberse al tamaño de la muestra y a las diferencias existentes entre los jugadores de baloncesto dependiendo de la posición.

La principal fortaleza de este estudio radica en la aportación de información acerca de los cambios en las variables antropométricas que se producen en la pretemporada en jugadores profesionales de baloncesto, habiéndose encontrado pocos estudios previos que hagan alusión a estas cuestiones. La principal limitación del estudio es el tamaño de la muestra, así como el tiempo transcurrido entre mediciones y la ausencia de un grupo control. Estas limitaciones abren camino a futuros trabajos en esta línea de investigación, que pasan por la inclusión de muestras mayores y análisis de las variables antropométricas de los jugadores en distintos momentos de la temporada, no solo en la pretemporada, para evaluar cómo se modifican durante su transcurso.

\section{Conclusiones}

Catorce días de pretemporada son suficientes para provocar cambios en algunos parámetros antropométricos, sobre todo los relacionados con la adiposidad, así como en la composición corporal, más concretamente con la masa grasa y muscular. Estos cambios acercan la morfología de jugador a lo establecido como ideal para este deporte.

\section{Referencias}

Abella del Campo, M., Escortell, R., Sospedra, I., Norte-Navarro, A., Martínez-Rodríguez, A., \& Martinez-Sanz, J. (2015). Características cineantropométricas en jugadores de baloncesto adolescentes. Revista Española de Nutrición Humana y Dietética, 20(1), 23-31. http:// dx.doi.org/10.14306/renhyd.20.1.179

Cabañas, M., Maestre, M., \& Herrero, A. (2008). Estudio de dos propuestas sobre el modelo «phantom» de proporcionalidad de Ross y Wilson. Biomecánica, 16(1), 7-12.

Campos, F., González-Víllora, S., González-Gómez, D., \& Martins, F. (2018). Benefits of 8-week fitness programs on health and fitness parameters (Beneficios de los programas de fitness de ocho semanas en parámetros de salud y fitness). Retos, 35, 224-228.

Carling, C. \& Orhant, E. (2010). Variation in Body Composition in Professional Soccer Players: Interseasonal and Intraseasonal Changes and the Effects of Exposure Time and Player Position. Journal of Strength and Conditioning Research, 24(5), 1332-1339.

Carter, J. E. L. \& Heath, B. H. (1990). Somatotyping: development and application. Cambridge: Cambridge University Press.

Cohen J. (1988). Statistical power analysis for the behavioral science. 2nd ed. Hillsdale, NJ: Lawrence Erlbaum Associates.

Costa, I. A. (2005). Características Físico-Fisiológicas de los Jugadores de Basquetbol. G-SE.COM, 729, 1-7.

De Rose, E. H. \& Guimaraes, A. C. (1980). A model for optimization of somatotype in young athletes. En M. Ostyn, G. Buenen, \& J. Simons (Eds). Kinanthropometry II. Baltimore: University Park Press

Del Pozo, J., Olcina, G., Brazo, J., Robles, C., \& Muñoz, D. (2009). Valoración de la composición corporal en jovenes jugadores de baloncesto y diferencias entre categorías de las selecciones territoriales de extremadura. Cuadernos de Psicología del Deporte, 9(Suppl.), 77.

Esparza-Ros, F. (1993). Manual de Cineantropometría. Madrid: Grupo Español de Cineantropometría.

Fuke, K., Dal Pupo, J., \& Corrêa, S. (2009). Evaluación de la composición corporal y de la flexibilidad en futbolistas profesionales en diferentes etapas del ciclo de entrenamiento. Archivos de Medicina del Deporte, 129(26), 7-13.

Garthe, I., Raastad, T., Refsnes, P. E., Koivisto, A., \& SundgotBorgen, J. (2011). Effect of two different weight-loss rates on body composition and strength and power-related performance in elite athletes. International Journal of Sport Nutrition and Exercise Metabolism, 21(2), 97-104.

Gaurav, V., Singh, M., \& Singh, S. (2010). Anthropometric characteristics, somatotyping and body composition of volleyball and basketball players. Journal of Physical Education and Sports Management, 1(3), 28-32.

Guedes, D. (2012). Clinical procedures used for analysis of the body composition. Brazilian Journal of Kinanthropometry and Human Performance, 15(1), 113129.

Godoy-Cumillaf,A. E., Cárcamo-Araneda, C. R., HermosillaRodríguez, F. P., Oyarzún-Ruíz, J. P., \& Viveros-Herrera, J. 
F. (2015). Estado nutricinal mediante parámetros antropométricos y bioquímicos de basquetbolistas universitarias. Nutrición Hospitalaria, 32(6), 2828-2831.

Helms, E. R., Aragon, A. A., \& Fitschen, P. J. (2014). Evidencebased recommendations for natural bodybuilding contest preparation: nutrition and supplementation. Journal of the International Society of Sports Nutrition, 11(20).

Hoare, D. (2000). Predicting success in junior elite basketball players - the contribution of anthropometic and physiological attributes. Journal of Science and Medicine in Sport, 3(4), 391-405.

Hoffman, M. D., Lebus, D. K., Ganong, A. C., Casazza, G. A., \& Loan, M. (2010). Body composition of 161-km ultramarathoners. International Journal of Sports Medicine, 31, 106-109.

Jelicic, M., Sekulic, D., \& Marinovic, M. (2002). Anthropometric characteristics of high level European junior basketball players. Collegium Antropologicum, 26, 69-76.

Latorre, P. A., Salas, J., \& Soto-Hermoso, V. M. (2012). Composición corporal relacionada con la salud en atletas veteranos. Nutrición Hospitalaria, 27(4), 1236-1243.

Matiegka, J. (1921). The testing of physical efficiency. American Journal of Physical Anthropology, 4, 223-230.

Ostojic, S. (2003). Seasonal alterations in body composition and sprint performance of elite soccer players. Journal of Exercise Physiology Online, 6(3), 24-27.

Popovic, S., Akpinar, S., Jaksic, D., Matic, R., \& Bjelica, D. (2013) Comparative study of anthropometric measurement and body composition between elite soccer and basketball players. International Journal of Morphology, 31(2), 461-467.

Puentes, J. \& Díaz, R. E. (2016). Correlación entre los factores antropométricos y la fuerza explosiva del tren inferior en jugadores jóvenes de baloncesto masculino. Santo Tomas: Universidad de Santo Tomás, Chile.

Ramos, D., Rubio, J., Martínez, F., Esteban, P., \& Jiménez, J. (2010). Características fisiológicas, podológicas y somatométricas del jugador profesional de baloncesto. Archivos de Medicina del Deporte, 27(136), 84-94.

Rivera-Sosa, J. (2016). Propiedades Antropométricas y Somatotipo de Jugadores de Baloncesto de Diferente Nivel Competitivo. International Journal of Morphology, 34(1), 179-188.

Rivera-Sosa, J., Martínez, C. P., \& Ortiz-Gómez, O. R. (2017). Perfil antropométrico y de proporcionalidad en jugadores de baloncesto mexicano. Revista Mexicana de Investigación en Cultura Física y Deporte, 6(8), 18-34.
Rocha, M. S. L. (1975). Peso ósseo do brasileiro de ambos os sexos de 17 a 25 años. Argentina Anatomía Antropológica, 1, 445-451.

Ross W. D. \& Wilson N. C. (1974). A stratagem for proportional growth assessment. Children in Exercise. ACTA Paediatrica Belgica, 28(Suppl), 169-182.

Siders, W. A., Bolonchuk, W. W., \& Lukaski, H. C. (1991) Effects of participation in a collegiate sport season on body composition. Journal of Sports Medicine and Physical Fitness, 31, 571-576.

Stewart, A., Marfell-Jones, M., Olds, T., \& de Ridder, H. (2011). International standards for anthropometric assessment. Portsmouth: International Society for Advancement in Kinanthropometry.

Tárrega L. \& Canda, A. (2010). Composición corporal del jugador de baloncesto. En F. Drobnic, J. Puigdellívol, \& T. Bové (Eds.). Bases científicas para la salud y un óptimo rendimiento en baloncesto. Madrid: Ergón.

Terry, R. B., Wood, P. D., Haskell, W. L., Stefanick, M. L., \& Krauss, R. M. (1989). Regional adiposity patterns in relation to lipids, lipoprotein cholesterol, and lipoprotein subfractions mass in men. Journal of Clinical Endocrinology and Metabolism, 68(1), 191-199.

Torres-Unda, J., Zarrazquin, I., Gil, J., Ruiz, F., Irazusta, A., Kortajarena, M., Seco, J., \& Irazusta, J. (2013). Anthropometric, physiological and maturational characteristics in selected elite and non-elite male adolescent basketball players. Journal of Sports Sciences, 31(2), 196-203.

Vaquera, A., Santos, S., Villa, J. G., Morante, J. C., \& GarcíaTormo, V. (2015). Anthropometric characteristics of Spanish professional basketball players. Journal of Human Kinetics, 46, 99-106.

Vargas, M., Lancheros, L., \& Barrera, M. (2011). Energy expenditure in repose related to body composition in adults. Revista de la Facultad de Medicina, 59, 43-58.

Würch, A. (1974). La femme et le sport. Médecine Sport Francais, 4, 441-445.

Yuhasz, M. S. (1974). Physical Fitness Manual. Ontario: West Ontario University.

Ziv, G \& Lidor, R. (2009) Physical attributes, physiological characteristics, on-court performances and nutritional strategies of female and male basketball players. Sports Medicine, 39(7), 547-568.

Zúñiga-Galaviz, U., Osorio-Gutiérrez,A., Toledo-Domínguez, I., \& Herrera-Perea, R. (2017). Somatotipo en futbolistas mexicanos profesionales de diferente nivel competitivo (Somatotype of Mexican soccer players from different competition level). Retos, 34, 100-102.

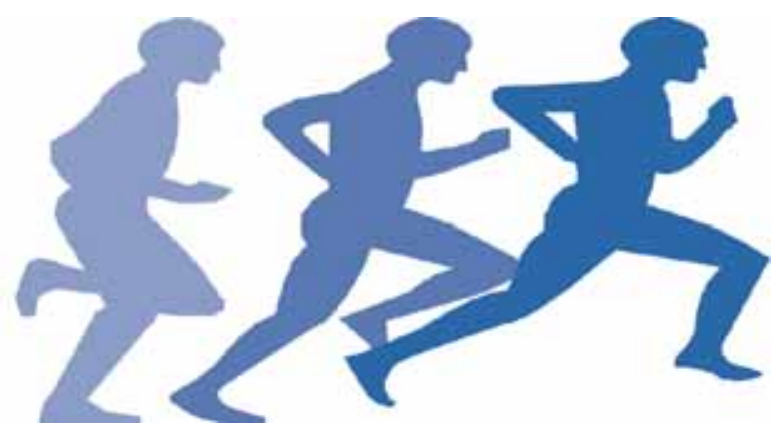

